\title{
Expression of Angiogenic Molecules in Cochlear Vasculature
}

\author{
Changjoon Lee, Hyong-Ho Cho, Sungsu Lee \\ Department of Otolaryngology-Head and Neck Surgery, Chonnam National University Hospital, Gwang-ju, Republic of Korea
}

\section{Email address:}

minsunglss@naver.com (Sungsu Lee)

\section{To cite this article:}

Changjoon Lee, Hyong-Ho Cho, Sungsu Lee. Expression of Angiogenic Molecules in Cochlear Vasculature. American Journal of Clinical and Experimental Medicine. Vol. 3, No. 5, 2015, pp. 279-282. doi: 10.11648/j.ajcem.20150305.24

\begin{abstract}
An altered vascular function may be related with several inner ear diseases such as Meniere's disease, sudden deafness, and noise-induced hearing loss. The present study was aimed to visualize the cochlear blood-labyrinth barrier and to analyze angiogenic molecules in the cochlear vasculature. Murine cochlea was obtained and its bony shell was removed. Whole mount immunostaining of endothelial cell markers, PECAM-1 and VE-cadherin, was done. There were pericytes and vascular smooth muscle cells shown. Angiogenic molecules including VEGFR2, VEGFR3, Sox17 and Dll4 were expressed. Precapillary arterioles, stria vascularis, and postcapillary venules were shown in the cochlear vasculature. The components of blood-labyrinth barrier were observed from basal turn to apical turn of the cochlea. The endothelial expression of VEGFR3, VEGFR2, and Sox17 denotes that the cochlea is not in a static, but in an active state. The robust expression of claudin-5 suggests its important role in blood-labyrinth barrier. The expression of a-SMA represents its need for vascular contraction. Visualizing cochlear vessels and determining angiogenic molecules could help to understand the pathophysiology of hearing loss ailments.
\end{abstract}

Keywords: Hearing Loss, Cochlea, Vasculature, Angiogenesis

\section{Introduction}

Hearing loss is one of the most common diseases, and is ever more increasing especially in the aged. There have been no definitive treatment regimens against hearing impairment, so that a patient should rely on a device like hearing aid or cochlear implantation.

In auditory sensory hair cells, sound signal is converted to electrical signal and propagated through the nerve to the brain. An impaired hair cell function should play a key pathophysiological role in hearing loss. Although there have been several trials to regenerate the auditory hair cell [1] [2], the cellular regeneration and subsequent maintenance have been poor.

An impairment of the cochlear vasculature has been also suggested to play an important role in several inner ear diseases such as Meniere's disease [3] [4], presbycusis [5] [6], sudden deafness [7] [8], and noise-induced hearing loss [9]. However, little has been known on the characteristics and functions of the cochlear vasculature. The present study was aimed to visualize the cochlear blood-labyrinth barrier and to determine the expression of angiogenic molecules in cochlear vessels.

\section{Materials and Methods}

\subsection{Animal Models}

C57BL/6 (Jackson Lab) and ICR mice were used. Eight-week old mice were used for the adult period, and postnatal day 5 pups were used for the neonatal period. To determine the expression of Sox17, we used knock-in mice that had GFP signal in the Sox17 locus [10]. The animals were maintained according the Guideline of Institutional Research Animal Committee.

\subsection{Immunohistochemistry}

\subsubsection{Tissue Harvest for Immunohistochemistry}

The animals were euthanized with intraperitoneal injection of high dose ketamine and xylazine. Temporal bones were collected. After removal of the bulla, 2 4\% paraformaldehyde was perfused through the round window. The sample was further fixed at $4^{\circ} \mathrm{C}$ for $2 \sim 10$ hours. In the adult mouse, it was then decalcified in $10 \%$ EDTA for $2-3$ days. Cochlear turns were dissected for whole mount. For cryosection, the tissue was sequentially dehydrated in $10 \%$, $20 \%, 30 \%$ sucrose, embedded in OCT compound, and frozen 
into a block. Cryosection was done with Leica cryotome in $10 \mu \mathrm{m}$ thickness.

\subsubsection{Immunohistochemistry Protocol}

Samples were perfused in $0.1 \%$ PBST and blocked with $5 \%$ donkey serum for 1 hour. Primary antibodies were treated in the blocking solution, at $4{ }^{\circ} \mathrm{C}$ overnight. Samples were washed 3 times with $0.1 \%$ PBST. Secondary antibodies were incubated according to the primary antibodies at room temperature for 2-4 hours. Final whole mounted tissues or sectioned slices were observed with confocal microscope (Zeiss LSM510)

\subsubsection{Antibodies for Immunohistochemistry}

Anti-VEGF antibody (abcam 46154), anti-mouse PECAM antibody(Chemicon, clone 2H8), anti-mouse VE-cadherin antibody(BD Pharmingen, clone 11D4.1), anti-VEGFR2 antibody(BD, clone Avas12), anti-angiopoietin-2 antibody(ab8452), anti-D114 antibody(ab7280), anti-Sox17 antibody(R\&D, AF1924), anti-aSMA antibody(Sigma, clone 1A4), anti-NG2 antibody(Millipore, AB5320), anti-claudin 5(Abcam, ab53765), Phalloidin (Invitrogen)

\section{Results and Discussion}

\subsection{Visualization of the Cochlear Vasculature}

Using the endothelial marker, PECAM1, precapillary arterioles, capillaries, and postcapillary venules were visualized (Figure 1). The expression of E-cadherin was pronounced in the lateral wall especially at the stria vascularis (Figure 2). The robust expression of smooth muscle actin suggests its active work making the endolymph. Cochlea is a well-organized structure in terms of the vascular formation.

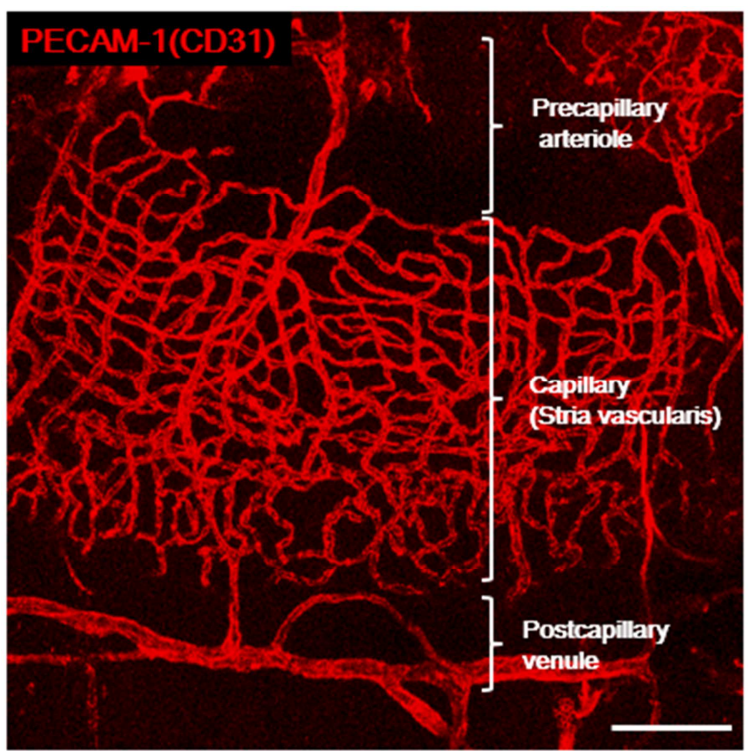

Figure 1. Visualization of the cochlear vasculature, represented by an endothelial marker PECAM-1. Precapillary arterioles, capillaries and postcapillary venules were well seen in an organized structure. Scale bar: $100 \mu \mathrm{m}$.
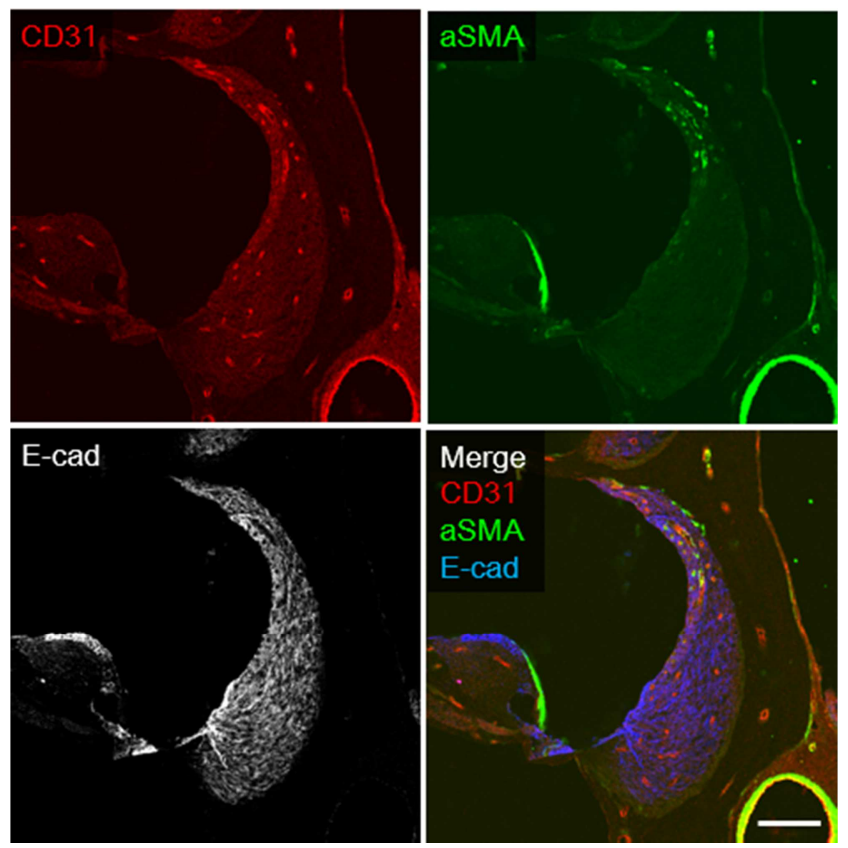

Figure 2. Visualization of the lateral wall where the vasculature is especially abundant and well organized. Epithelial marker, E-cadherin, is seen at the stria vascularis and the spiral ligament. Vessels are concentrated in the stria vascularis (capillary) where the endolymph is made. Note the robust expression of CD31 and a-SMA in the stria vascularis, representing the need of the high amount of blood flow. Scale bar: $100 \mu \mathrm{m}$.

\subsection{Visualization of the Blood-Labyrinth Barrier in the Cochlear Vasculature}

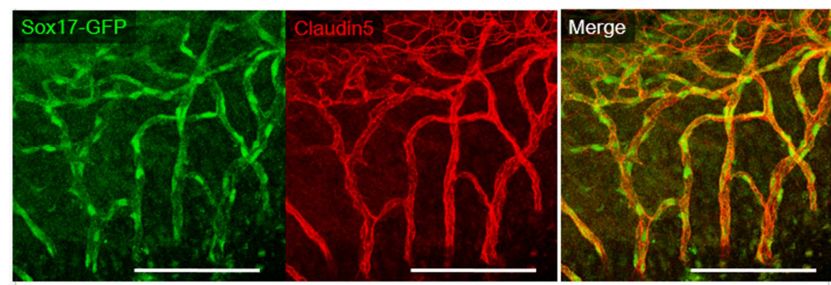

Figure 3. Claudin-5, was highly expressed in the entire cochlear vessels. Sox17 (GFP) was used to demonstrate the endothelial cell. Note the coexpression of both molecules. Scale bar: $100 \mu \mathrm{m}$.

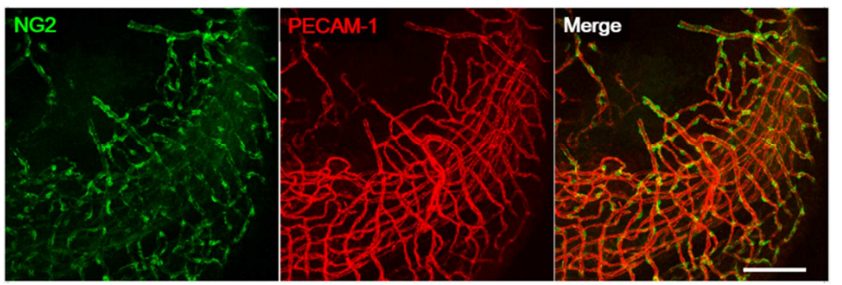

Figure 4. Pericytes were stained with NG2. The cochlear vasculature robustly expressed NG2. NG2-positive pericytes well covered the endothelial cell shown by PECAM-1. Scale bar: $100 \mu \mathrm{m}$.

As with other cranial structures, the cochlea is protected from the circulatory system through blood-labyrinth barrier. Three major cellular components of the blood-labyrinth barrier are endothelial cells, fibrocysts and pericytes. The endothelial cells have a unique structure called tight-junction, which is important to prevent potential damage to the structure from various circulating toxic substances. Claudin-5 
is a marker that is specifically seen in the endothelial cell. Cochlea robustly expressed claudin-5 in the endothelial cell (Figure 3). Moreover, all the cochlear vessels were well covered with pericytes, demonstrating tight barrier function in the cochlea (Figure 4).

\subsection{Expression of Angiogenic Molecules in the Cochlear Vasculature}

While some vessels are in a stable and calm state, others may be in an active status. Vessels are newly made, or they may be regressed according to the need of the tissue. VEGF-VEGF receptor 2 signalling is the most powerful driving force in angiogenesis [11]. The expression of VEGF receptor 2 is upregulated in newly-formed vessels and downregulated in stable vessels. The robust expression of VEGF receptor 2 in cochlear vessels (Figure 5) suggests that the cochlea is in an active state.

Dll4 is one of the ligand for Notch receptor that is specifically expressed in the endothelial cell. It is expressed in active vessels especially in the tip cell which is the leading cellular component of the newly-forming vessels [12]. Sox17 also plays an important role in sprouting angiogenesis, being highly expressed in the tip cell. Cochlear vessels highly expressed both D114 and Sox17 (Figure 6).
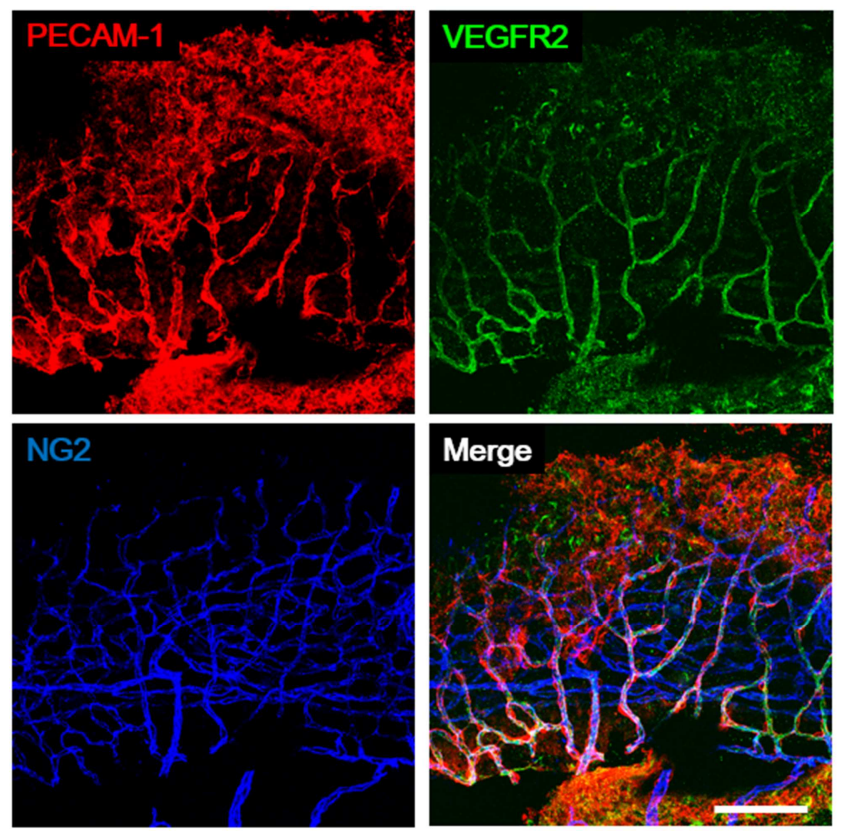

Figure 5. The most powerful angiogenic molecule VEGF receptor 2 (VEGFR2) was well expressed in cochlear endothelial cells, co-stained with PECAM-1. Pericyte marker NG2 is also well shown the vasculature. Scale bar: $100 \mu \mathrm{m}$.

VEGF receptors 3 (VEGFR3) were originally reported to play an important role in lymphatic vessels. However, they are also known to be expressed in active angiogenic blood vessels [13] [14]. They were highly expressed in the endothelial cells.

Cochlear formation is known to be finished at the time of the birth. Therefore, all these angiogenic molecules may not necessarily mean that the cochlea is making new vessels. Indeed, any tip cell formation was not observed at all. However, the cochlea seems to be in an active condition, suggesting that it is not a mere conduit for the hemopoietic cells. The precise role of these molecules in the cochlea remains to be further studied.

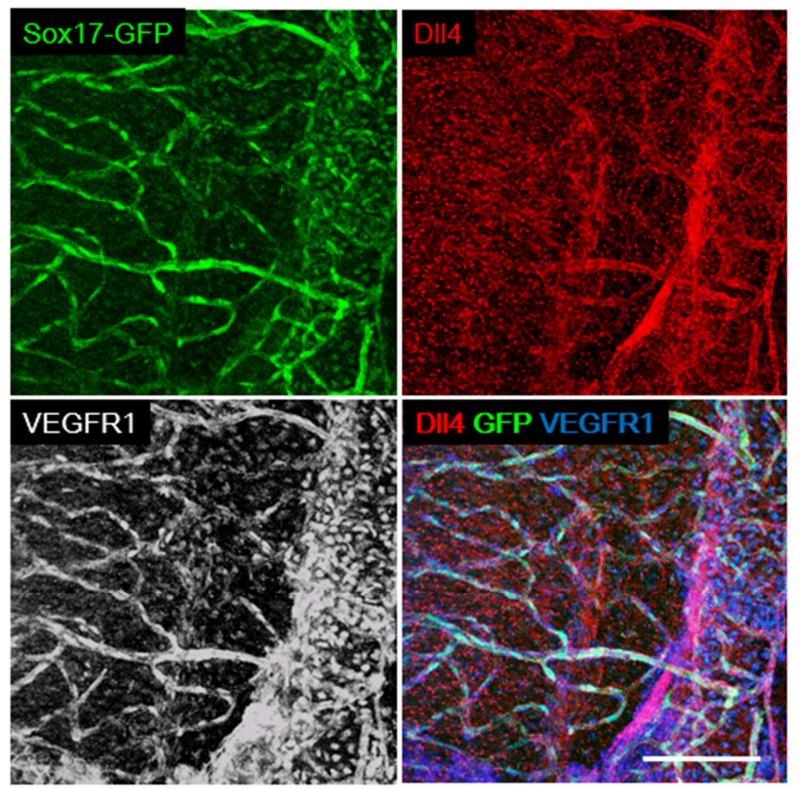

Figure 6. Tip cell markers, Dll4 and Sox 17, are highly expressed in cochlear vessels. VEGF receptor 1 (VEGFR1) is also well expressed in the entire cochlea. Scale bar: $100 \mu \mathrm{m}$.

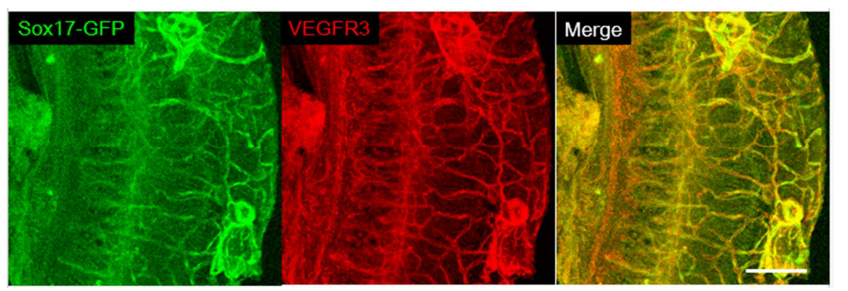

Figure 7. VEGF receptor 3 (VEGFR3) is highly expressed, co-stained with Sox17. Although VEGFR3 was origianlly a lymphatic vessel marker, it is well expressed in angiogenic vessels. This again suggests that the cochlea is in an active status. Scale bar: $100 \mu \mathrm{m}$.

\section{Conclusion}

Vessels are not merely a conduit for blood flow. It is newly made or regressed. It permits or limits many molecules on crossing over to the tissue. Cochlear vessels highly expressed angiogenic molecules, such as VEGFR2, VEGFR3, Sox17 and Dll4, demonstrating that they are in an active state. They had abundant tight-junctions and pericytes, suggesting a barrier function. Further studies on the function of the cochlea vasculature may give a new pathophysiological insight of the inner ear diseases.

\section{Acknowledgements}

This research was supported by Korea Research Foundation (2014R1A1A1036856). 


\section{References}

[1] K.R. Koehler, A.M. Mikosz, A.I. Molosh, D. Patel, E. Hashino, "Generation of inner ear sensory epithelia from pluripotent stem cells in 3D culture." Nature, 2013, 500(7461), pp. 217-221.

[2] K. Mizutari, M. Fujioka, M. Hosoya, N. Bramhall, H.J. Okano, H. Okano, A.S.B. Edge, "Notch inhibition induces cochlear hair cell regeneration and recovery of hearing after acoustic trauma," Neuron, 2013, Vol. 77, pp.58-69.

[3] J.P. Vasama, F.H. Linthicum, "Meniere's disease and endolymphatic hydrops without Meniere's symptoms: temporal bone histopathology," Acta oto-laryngologica, 1999, vol. 119, pp.297-301.

[4] F. Ihler, M. Bertlich, K. Sharaf, S. Strieth, M. Strupp, M. Canis, "Betahistine exerts a dose-dependent effect on cochlear stria vascularis blood flow in guinea pigs in vivo," PloS one, 2012, vol.7, pp.e39086.

[5] E.G. Nelson, R. Hinojosa, "Presbycusis: a human temporal bone study of individuals with downward sloping audiometric patterns of hearing loss and review of the literature," The Laryngoscope, 2006, vol. 116, pp.1-12.

[6] J.N. Brown, J.M. Miller, A.L. Nuttal, "Age-related changes in cochlear vascular conductance in mice," Hearing research, 1995, vol. 86, pp.189-194.

[7] M. M. Ciccone, F. Cortese, M. Pinto, "Endothelial function and cardiovascular risk in patients with idiopathic sudden sensorineural hearing loss," Atherosclerosis, 2012, vol.225, pp.511-516.

[8] C. Aimoni, C. Bianchini, M. Borin, "Diabetes, cardiovascular risk factors and idiopathic sudden sensorineural hearing loss: a case-control study," Audiology \& neuro-otology, 2010, vol.15, pp.111-115.

[9] W. Arpornchayanon, M. Canis, M. Suckfuell, F. Ihler, B. Olzowy, S. Strieth, "Modeling the measurements of cochlear microcirculation and hearing function after loud noise," Otolaryngology--head and neck surgery, 2011, vol.145, pp.463-469.

[10] S.H. Lee, S. Lee, H. Yang, S. Song, K. Kim, T.L. Saunders, J.K. Yoon, G.Y. Koh, I. Kim, Circulation Research, 2014, Vol115(2), pp.215-226.

[11] N. Ferrara, H.P. Gerber, J. LeCouter, "The biology of VEGF and its receptors," Nature Medicine, 2003, vol.9(6), pp.669-676.

[12] M. Hellstrom, L.K. Phng, J.J. Hofmann, E. Wallgard, L. Coultas, P. Lindblom, J. Alva, A.K. Nilsson, L. Karlsson, N. Gaiano, K. Yoon, J. Rossant, M.K. Iruela-Arispe, M. Kalén, H. Gerhardt, C. Betsholtz, "Dl14 signalling through Notch1 regulates formation of tip cells during angiogenesis, "Nature, 2007, vol445(7129), pp.776-780.

[13] T. Tammela, G. Zarkada, H. Nurmi, L. Jakobsson, K. Heinolainen, D. Tvorogov, W. Zheng, C.A. Franco, A. Murtomäki, E. Aranda, N. Miura, S. Ylä-Herttuala, M. Fruttiger, T. Mäkinen, A. Eichmann, J.W. Pollard, H. Gerhardt, K. Alitalo.

[14] R. Benedito, S.F. Rocha, M. Zamykal, F. Radtke, O. Casanovas, A. Duarte, B. Pytowski, R.H. Adams, "Notch-dependent VEGFR3 upregulation allows angiogenesis without VEGF-VEGFR2 signalling,” Nature, 2012, vol.484(7392), pp.110-114. 